\title{
EXISTENCE OF LIMIT CYCLES IN A PREDATOR-PREY SYSTEM WITH A FUNCTIONAL RESPONSE
}

\author{
BASEM S. ATTILI
}

(Received 16 January 2001)

\begin{abstract}
We consider the existence of limit cycles for a predator-prey system with a functional response. The system has two or more parameters that represent the intrinsic rate of the predator population. A necessary and sufficient condition for the uniqueness of limit cycles in this system is presented. Such result will usually lead to a bifurcation curve.
\end{abstract}

2000 Mathematics Subject Classification. 92D40.

1. Introduction. The interaction of a predator-prey populations in its simplest format can be modeled by a system of differential equations of the form

$$
\frac{d x}{d t}=c_{1} x-k_{1} x y, \quad \frac{d y}{d t}=-c_{2} y-k_{2} x y,
$$

together with some initial conditions $x=x_{0}$ and $y=y_{0}$ at time $t=0$. Where $x$ and $y$ represent the sizes of the competing populations at any given time, $c_{1}, c_{2}, k_{1}$, and $k_{2}$ are positive constants. The constants $c_{1}$ and $c_{2}$ relates the growth (decay) of the prey (predator) populations. It is logical to assume that the number of encounters of predator and prey is proportional to $x y$ and this justifies the presence of such terms in both equations of (1.1), see Simmons [5].

We consider a more general system than (1.1). Throughout, the predator-prey system we are going to use is of the form

$$
\frac{d x}{d t}=r x(1-x)-y \theta(x), \quad \frac{d y}{d t}=-D y+s y \theta(x),
$$

where $x$ and $y$ are the prey and the predator population sizes, respectively, $\theta(x)$ is a functional response of predator while $r, s$, and $D$ are positive parameters. We also assume

$$
\begin{gathered}
\theta(0)=0, \\
\theta^{\prime}(x)>0 \text { for } x \geq 0, \quad \theta^{\prime \prime}(x)<0, \quad \theta^{\prime \prime \prime}(x)>0 \quad \text { for } x>0, \\
\lim _{x \rightarrow \infty} \theta(x)=k, \quad k \text { is finite. }
\end{gathered}
$$

We investigate system (1.2) under the assumptions (1.3). In particular, we try to give a necessary and sufficient condition for the nonexistence of limit cycles of (1.2).

The problem of existence and uniqueness of limit cycles have been under investigation recently, for example, Kuang and Freedman [4] considered the question of uniqueness of limitcycles in Gauss type models, Kooj and Zegling [3] considered a 
system with Ivlev functional response; that is, $\theta(x)=1-e^{-a x}$ where $a$ is a positive parameter, Sugie [6] considered the two parameter bifurcation for systems with Ivlev type response.

The outline of this paper is as follows. In Section 2, we derive the necessary condition for the nonexistence of limit cycles. In Section 3, we present the sufficient condition for the nonexistence of such cycles. Finally, in Section 4, we give some models from ecology that fits the framework of our setup we are presenting.

2. Necessary conditions for the absence of limit cycles. We start this section by studying system (1.2). By putting $d x / d t=P$ and $d y / d t=Q$, the system $P=Q=0$ has a critical (singular) point $\left(x^{*}, y^{*}\right)$ such that $y^{*}\left(s \theta\left(x^{*}\right)-D\right)=0$ which leads to $\theta\left(x^{*}\right)=D / s$ and $r x^{*}\left(1-x^{*}\right)-y^{*} \theta\left(x^{*}\right)=r x^{*}\left(1-x^{*}\right)-y^{*} D / s=0$ or equivalently $y^{*}=r s x^{*}\left(1-x^{*}\right) / D$. If $x^{*} \in(0,1)$ and $0<D / s<k$, then the critical point $\left(x^{*}, y^{*}\right)$ will be in the first quadrant, while if the condition $0<D / s<k$ is not satisfied, there will be no critical points in the first quadrant and hence no limit cycles of (1.2) exist.

By inspecting system (1.2) also, it is clear that $(0,0)$ and $(1,0)$ are critical points and if one considers the linearized form of (1.2), then both points are saddle points. Now consider the following result.

LEMMA 2.1. Assume (1.3) holds, then system (1.2) has no limit cycles if and only if

$$
r\left(2 x^{*}-1\right)+y^{*} \theta^{\prime}\left(x^{*}\right) \geq 0 .
$$

This lemma is a modification of Theorem 3.1 of Sugie and Hara [7] and Theorem 3.1 of Sugie [6] and for details one can refer to them. Now define

$$
\Omega=\left\{(x, y) \in \mathbb{R}_{+}^{2} ; 0 \leq x \leq 1,0 \leq \frac{s x+y}{s} \leq \frac{s+M}{s}\right\},
$$

where

$$
M=\max \{r x(1-x) ; 0 \leq x \leq 1\} .
$$

With this we will present a result that guarantees the boundedness of the solution of (1.2) in the first quadrant, for details on similar results refer to Freedman and Hso [2].

THEOREM 2.2. For $\left(x_{0}, y_{0}\right) \in \mathbb{R}_{+}^{2}$, then $(x(t), y(t)) \rightarrow(x, y) \in \Omega$ as $t \rightarrow \infty$ and the set $\Omega$ is positively invariant.

Rewriting the system (1.2) in the form

$$
\dot{X}=F(X),
$$

where $X=(x, y)^{T}$ and $F=\left(F_{1}, F_{2}\right)^{T}=[r x(1-x)-y \theta(x),-D y+s y \theta(x)]^{T}$ and linearizing about $\left(x^{*}, y^{*}\right)$, one obtains

$$
L=\left[\begin{array}{cc}
r-2 r x^{*}-y^{*} \frac{d \theta}{d x}\left(x^{*}\right) & -\theta\left(x^{*}\right) \\
y^{*} s \frac{d \theta}{d x}\left(x^{*}\right) & s \theta\left(x^{*}\right)-D
\end{array}\right],
$$


with $\theta\left(x^{*}\right)=D / s$ as was established earlier and replacing $d \theta / d x$ by $\theta^{\prime}(x)$ the $L_{22}$ element will be zero. Hence $L$ becomes

$$
L=\left[\begin{array}{cc}
r-2 r x^{*}-y^{*} \theta^{\prime}\left(x^{*}\right) & -\theta\left(x^{*}\right) \\
y^{*} s \theta^{\prime}\left(x^{*}\right) & 0
\end{array}\right] .
$$

The characteristic polynomial for $L$ will then be

$$
P(\lambda)=\left[\left(r-2 r x^{*}-y^{*} \theta^{\prime}\left(x^{*}\right)\right)-\lambda\right] \lambda+y^{*} s \theta^{\prime}\left(x^{*}\right) \theta\left(x^{*}\right)=0
$$

or

$$
P(\lambda)=\lambda^{2}+\left[-r+2 r x^{*}+y^{*} \theta^{\prime}\left(x^{*}\right)\right] \lambda-y^{*} s \theta^{\prime}\left(x^{*}\right) \theta\left(x^{*}\right)=0
$$

with roots

$$
-\frac{\left[-r+2 r x^{*}+y^{*} \theta^{\prime}\left(x^{*}\right)\right] \pm \sqrt{\left[-r+2 r x^{*}+y^{*} \theta^{\prime}\left(x^{*}\right)\right]^{2}+4 y^{*} s \theta^{\prime}\left(x^{*}\right) \theta\left(x^{*}\right)}}{2} .
$$

The term under the radical sign is positive since by assumption we have $s>0, y^{*} \in \Omega$, $\theta^{*}\left(x^{*}\right)=D / s$ where $D, s>0$ and $\theta^{\prime}>0$ by assumption (1.3). This means that to have positive roots, we must have

$$
2 r x^{*}+y^{*} \theta^{\prime}\left(x^{*}\right)-r<0 .
$$

As a result, $\left(x^{*}, y^{*}\right)$ will be unstable if (2.10) is satisfied. This means from Theorem 2.2 and Poincaré-Bendixon theorem, see Coddington and Levinson [1], that each orbit starting at a point in the first quadrant is a limit cycle. This leads to the following result.

Proposition 2.3. If system (1.2) has no limit cycles, then

$$
2 r x^{*}+y \theta^{\prime}\left(x^{*}\right)-r \geq 0 .
$$

Thus obtaining the necessary condition for the nonexistence of limit cycles. For the other critical points; namely, $(0,0)$ and $(1,0)$, the stable manifold for $(0,0)$ is at the $y$-axis while the unstable is at the $x$-axis. For $(1,0)$, the unstable manifold is in the first quadrant.

3. Transformation into a Liénard system and the sufficient condition. To establish the sufficient condition for the nonexistence of limit cycles, we start by transforming (1.2) into a Liénard system by introducing the change of variables

$$
u=x-x^{*}, \quad v=\log \frac{y}{y^{*}}, \quad d s=-\theta(x) d t
$$

where $\left(x^{*}, y^{*}\right)$ is the critical point in the first quadrant as mentioned before. 
Now using the chain rule; that is, $d u / d s=d x / d s=(d x / d t)(d t / d s)$ on the first part of (1.2) leads to

$$
\begin{aligned}
\frac{d u}{d s} & =(r x(1-x)-y \theta(x))\left(-\frac{1}{\theta(x)}\right) \\
& =\left[r x\left(u+x^{*}\right)\left(1-\left(u+x^{*}\right)\right)-y^{*} \theta\left(u+x^{*}\right)\right]\left(-\frac{1}{\theta\left(u+x^{*}\right)}\right) \\
& =-\left[\left(\frac{r\left(u+x^{*}\right)\left(1-\left(u+x^{*}\right)\right)}{\theta\left(u+x^{*}\right)}-y^{*}\right)+\left(y^{*} e^{v}-y^{*}\right)\right],
\end{aligned}
$$

or

$$
\frac{d u}{d s}=-f(u)+h(v)
$$

where

$$
f(u)=\frac{r\left(u+x^{*}\right)\left(1-\left(u+x^{*}\right)\right)}{\theta\left(u+x^{*}\right)}-y^{*}, \quad h(v)=y^{*} e^{v}-y^{*} .
$$

Considering the second part of (1.2), we have

$$
\frac{d v}{d s}=-\frac{1}{y} \frac{d y}{d s}=-\frac{1}{y} \frac{d y}{d t} \frac{d t}{d s}=\frac{1}{y \theta(x)} \frac{d y}{d t} .
$$

This leads to

$$
\frac{d v}{d s}=\frac{1}{y \theta(x)}[y s \theta(x)-y D]=s-\frac{D}{\theta\left(u+x^{*}\right)}
$$

or

$$
\frac{d v}{d s}=g(u), \quad g(u)=s-\frac{D}{\theta\left(u+x^{*}\right)} .
$$

Combining (3.3) and (3.7) leads to the system

$$
\frac{d u}{d s}=-f(u)+h(v), \quad \frac{d v}{d s}=g(u),
$$

where $f(u), h(v)$, and $g(u)$ are real-valued continuous functions defined on $I=$ $(-b, c)$ with $b, c>0$ and may be infinite. We examine the properties of these functions.

Since the critical point $\left(x^{*}, y^{*}\right)$ is such that $\theta\left(x^{*}\right)=D / s$ and $y^{*}=r s x^{*}\left(1-x^{*}\right) / D$ this means

$$
\begin{gathered}
f(0)=0, \quad g(0)=0, \quad h(0)=0, \\
u g(u)>0 \quad \text { if } u \neq 0, \quad v h(v)>0 \quad \text { if } v \neq 0, \\
f(u) \longrightarrow-\infty, \quad g(u) \longrightarrow \infty \quad \text { as } u \longrightarrow \infty .
\end{gathered}
$$

Also since $\theta^{\prime}>0$ and $D>0$, then $(d / d u)(g(u))=D \theta^{\prime}\left(u+x^{*}\right) /\left[\theta\left(u+x^{*}\right)\right]^{2}>0$ for $u \in I$ and $(d / d u)(h(v))=y^{*} e^{v}>0$ for $v \in \mathbb{R}$. This justifies (3.9). 
Now let

$$
G(u)=\int_{0}^{u} g(\xi) d \xi
$$

and define $\tau=G(u) \operatorname{sgn}(u)$, then consider the following property (stated in Theorem 3.1 of Sugie [6] and for the proof refer to [6]).

THEOREM 3.1. If $f(u), g(u)$, and $h(v)$ satisfy (3.9) and

$$
f\left(G^{-1}(-\tau)\right) \neq f\left(G^{-1}(\tau)\right) ; \quad 0<\tau<L,
$$

where $L=\min \{G(-b), G(c)\}$. Then the system (3.8) has no limit cycles in the set $\{(u, v) ; u \in I$ and $v \in \mathbb{R}\}$.

To show that (3.11) is satisfied for the setting in (3.8), we need some results on $f(u), g(u)$, and $h(v)$.

LEMMA 3.2. If $g(u)$ is as given in (3.8), then $G(-u)>G(u)$ for $u \in\left(0, x^{*}\right)$.

Proof. From (3.10), we have

$$
\frac{d}{d u}[G(-u)-G(u)]=\frac{\gamma(u)}{\theta\left(u+x^{*}\right) \theta\left(-u+x^{*}\right)} ; \quad u \in\left(0, x^{*}\right),
$$

where

$$
\gamma(u)=D\left[\theta\left(u+x^{*}\right)+\theta\left(-u+x^{*}\right)\right]-2 s \theta\left(u+x^{*}\right) \theta\left(-u+x^{*}\right) .
$$

Differentiating $\gamma(u)$, we obtain

$$
\begin{aligned}
\frac{d}{d u}(\gamma(u))= & \theta^{\prime}\left(u+x^{*}\right)\left[-s \theta\left(-u+x^{*}\right)+D\right]+\theta^{\prime}\left(-u+x^{*}\right)\left[s \theta\left(u+x^{*}\right)+D\right] \\
& +s\left[\theta\left(u+x^{*}\right) \theta^{\prime}\left(-u+x^{*}\right)-\theta^{\prime}\left(u+x^{*}\right) \theta\left(-u+x^{*}\right)\right] .
\end{aligned}
$$

Using assumption (1.3b) leads to $(d / d u)(\gamma(u))>0$. Now since $\lim _{u \rightarrow 0^{+}} \gamma(u)=0$, this implies $\gamma(u)>0$ for $u \in\left(0, x^{*}\right)$. Hence

$$
\frac{d}{d u}[G(-u)-G(u)]>0 ; \quad u \in\left(0, x^{*}\right) .
$$

Also since $\lim _{u \rightarrow 0^{+}}[G(-u)-G(u)]=0$, the conclusion of the lemma follows; that is, $G(-u)>G(u)$ for $u \in\left(0, x^{*}\right)$.

LEMMA 3.3. If (2.11) is satisfied, then $f(-u)>f(u)$ for $u \in\left(0, x^{*}\right)$.

Proof. Consider two cases $x^{*} \in(0,1 / 2]$ and $x^{*} \in(1 / 2,1)$. We consider the first case since the latter case can be done easily using a similar argument as that used in the proof of Lemma 3.2. We only prove the case $x^{*} \in(0,1 / 2]$. Consider $f(-u)-f(u)$ after adding and subtracting $y^{*} \theta^{\prime}\left(x^{*}\right) \theta\left(u+x^{*}\right) u$ and $y^{*} \theta^{\prime}\left(x^{*}\right) \theta\left(-u+x^{*}\right) u$, as a result we can write

$$
f(-u)-f(u)=\frac{\beta(u)+\alpha(u)}{\theta\left(u+x^{*}\right) \theta\left(-u+x^{*}\right)},
$$


where

$$
\begin{aligned}
& \beta(u)=\left[2 r x^{*}+y^{*} \theta^{\prime}\left(x^{*}\right)-r\right]\left(\theta\left(u+x^{*}\right)+\theta\left(-u+x^{*}\right)\right) u, \\
& \alpha(u)=y^{*} \theta\left(u+x^{*}\right)\left[\theta\left(x^{*}\right)-u \theta^{\prime}\left(x^{*}\right)\right]-y^{*} \theta\left(-u+x^{*}\right)\left[\theta\left(x^{*}\right)+u \theta^{\prime}\left(x^{*}\right)\right] .
\end{aligned}
$$

From assumptions (1.3) and (2.11), one can easily see that $\beta(u)>0$. For $\alpha(u)$ consider

$$
\begin{aligned}
\frac{d}{d u}(\alpha(u))= & y^{*} \theta^{\prime}\left(u+x^{*}\right)\left[\theta\left(x^{*}\right)-u \theta^{\prime}\left(x^{*}\right)\right]-y^{*} \theta^{\prime}\left(x^{*}\right) \theta\left(u+x^{*}\right) \\
& +y^{*} \theta^{\prime}\left(-u+x^{*}\right)\left[\theta\left(x^{*}\right)+u \theta^{\prime}\left(x^{*}\right)\right]-y^{*} \theta^{\prime}\left(x^{*}\right) \theta\left(-u+x^{*}\right), \\
\frac{d^{2}}{d u^{2}}(\alpha(u))= & y^{*} \theta^{\prime \prime}\left(u+x^{*}\right)\left[\theta\left(x^{*}\right)-u \theta^{\prime}\left(x^{*}\right)\right]-2 y^{*} \theta^{\prime}\left(x^{*}\right) \theta^{\prime}\left(u+x^{*}\right) \\
& -y^{*} \theta^{\prime \prime}\left(-u+x^{*}\right)\left[\theta\left(x^{*}\right)+u \theta^{\prime}\left(x^{*}\right)\right]+2 y^{*} \theta^{\prime}\left(x^{*}\right) \theta^{\prime}\left(-u+x^{*}\right) .
\end{aligned}
$$

Assumptions (1.3) implies that $\theta^{\prime}\left(-u+x^{*}\right)>\theta^{\prime}\left(u+x^{*}\right)$ and $\theta^{\prime \prime}\left(u+x^{*}\right)>\theta^{\prime \prime}(-u+$ $\left.x^{*}\right)$ for $u \in\left(0, x^{*}\right)$. This means that $\left(d^{2} / d u^{2}\right)(\alpha(u))>0$ for $u \in\left(0, x^{*}\right)$. Also since $\lim _{u \rightarrow 0^{+}}(d / d u)(\alpha(u))=0$, we obtain $(d / d u)(\alpha(u))>0 ; u \in\left(0, x^{*}\right)$. This leads to the conclusion that $\alpha(u)>0 ; u \in\left(0, x^{*}\right)$ since $\lim _{u \rightarrow 0^{+}} \alpha(u)=0$. Now since $\alpha(u)>0$ and $\beta(u)>0$ for $u \in\left(0, x^{*}\right)$ it follows that $f(-u)-f(u)>0$ for $u \in\left(0, x^{*}\right)$.

Now we need to study $f(u)$ and $f^{\prime}(u)$ in more details to be able to use Theorem 3.1. We will start by the following result.

LEMMA 3.4. The function $f^{\prime}(u)=0$ has at most one root if $u>-x^{*}$.

The proof involves some similar but longer arguments than the proof of Lemma 3.3 and it is omitted.

LEMMA 3.5. If (2.11) is satisfied, then

(1) If $f^{\prime}(u)=0$ has a root, it has to be negative and $f(u)$ has a local maximum at this root.

(2) If $f^{\prime}(u)=0$ has no root, then $f(u)$ is a decreasing function.

We will comment on the proof which will be done by contradiction. Consider

$$
f^{\prime}(u)=\frac{r\left[\left(1-2\left(u+x^{*}\right)\right) \theta\left(u+x^{*}\right)-\left(u+x^{*}\right)\left(1-\left(u+x^{*}\right)\right) \theta^{\prime}\left(u+x^{*}\right)\right]}{\theta^{2}\left(u+x^{*}\right)},
$$

one can see that $\lim _{u \rightarrow \infty} f^{\prime}(u)=-\infty$. Assume $f^{\prime}(u)=0$ has a positive root. Then since the limit of $f^{\prime}(u)$ is $-\infty$ as $x \rightarrow \infty$ and using (2.11), it follows that $f^{\prime}(u)=0$ must have another positive root. This is a contradiction to Lemma 3.4. Completing the proof of the first part. For the second part, condition (2.11) implies that $f^{\prime}(u)$ $\leq 0$. This means that $f(u)$ is decreasing on $I$.

Now consider

$$
\lim _{u \rightarrow-x^{*}} f(u)=\lim _{u \rightarrow-x^{*}} \frac{r\left(u+x^{*}\right)\left(1-\left(u+x^{*}\right)\right)}{\theta\left(u+x^{*}\right)}-y^{*} .
$$

Using l'Hospital's rule, we obtain

$$
\lim _{u \rightarrow-x^{*}} f(u)=\frac{r}{\theta^{\prime}(0)}-y^{*} .
$$


LEMMA 3.6. Assume (2.11) is satisfied. If $r / \theta^{\prime}(0)-y^{*}<0$, then $f^{\prime}(u)$ has a unique root for $-x^{*}<u<0$.

Proof. If $r / \theta^{\prime}(0)-y^{*}<0$, then by (2.11) there exists a $u^{*}$ such that $-x^{*}<u^{*}<0$ and $f(u)$ is increasing for $-x^{*}<u<u^{*}$ and decreasing for $u>u^{*}$. This proves the lemma.

LEMMA 3.7. If $f\left(-\lambda_{1}\right)=f\left(\lambda_{2}\right)$, then $\lambda_{1}>\lambda_{2}$.

Proof. With $G(u)$ as defined in (3.10), we have $G\left(-\lambda_{1}\right)=\tau=G\left(\lambda_{2}\right)$, where $\lambda_{1}=$ $-G^{-1}(-\tau), \lambda_{2}=G^{-1}(\tau)$, and $-x^{*}<\lambda_{1}<0<\lambda_{2}$. By Lemma 3.6, if $r / \theta^{\prime}(0)-y^{*}<$ 0 , then there exist a $u^{*} \in\left(-x^{*}, 0\right)$ such that $f^{\prime}\left(u^{*}\right)=0, f(u)$ is increasing for $u^{*} \in\left(-x^{*}, u\right)$ and decreasing for $u>u^{*}$. This means $-\lambda_{1}<u^{*}$ and $f\left(-\lambda_{1}\right)>$ $\lim _{u \rightarrow-x^{*}} f(u)=r / \theta^{\prime}(0)-y^{*}$. Now since $f\left(-\lambda_{1}\right)=f\left(\lambda_{2}\right)$, it follows that $f\left(\lambda_{2}\right)>$ $r / \theta^{\prime}(0)-y^{*}$. Thus from Lemma 3.3, we have $r / \theta^{\prime}(0)-y^{*}=\lim _{u \rightarrow-x} f f(u)>f\left(x^{*}\right)$ and $f\left(\lambda_{2}\right)>f\left(x^{*}\right)$. Since $f(u)$ is decreasing for $u>u^{*}$. This means $\lambda_{2}<x^{*}$, while $f\left(\lambda_{2}\right)=f\left(-\lambda_{1}\right)>f\left(\lambda_{1}\right)$. As a result, we have $\lambda_{1}>\lambda_{2}$. In exactly a similar way $\lambda_{1}>\lambda_{2}$ if $r / \theta^{\prime}(0)-y^{*}>0$ and $f^{\prime}(u)$ has a unique root. This completes the proof of the lemma.

Now with these results in Lemmas 3.2, 3.3, 3.4, 3.5, 3.6, and 3.7, we can prove the sufficient condition (3.11) of Theorem 3.1; that is,

$$
f\left(G^{-1}(-\tau)\right) \neq f\left(G^{-1}(\tau)\right) ; \quad 0<\tau<M .
$$

We do that by contradiction. For that reason, assume that there is a $\tau>0$ such that

$$
f\left(G^{-1}(-\tau)\right)=f\left(G^{-1}(\tau)\right) .
$$

Then

$$
\begin{gathered}
G\left(-\lambda_{1}\right)=\tau=G\left(\lambda_{2}\right), \\
f\left(-\lambda_{1}\right)=f\left(\lambda_{2}\right) .
\end{gathered}
$$

From Lemma 3.2, $G\left(-\lambda_{1}\right)>G\left(\lambda_{1}\right)$ while from (3.24) $G\left(\lambda_{2}\right)>G\left(\lambda_{1}\right)$. Now since $G(u)$ is increasing for $u>0$, we must have $\lambda_{2}>\lambda_{1}$. This is a contradiction to Lemma 3.7. Therefore (3.11) is satisfied.

4. Models from ecology. In this section, we present some predator-prey models that fit the frame work we presented in previous sections. We start with the simplest model.

MODEL 4.1. Lynxes and hares: the model has the form

$$
\frac{d x}{d t}=2 x-0.001 x y, \quad \frac{d y}{d t}=-10 y+0.002 x y,
$$

with an initial population of 5000 hares and 100 lynxes. The behavior of the recovery of each species is illustrated graphically in Figure 4.1. 


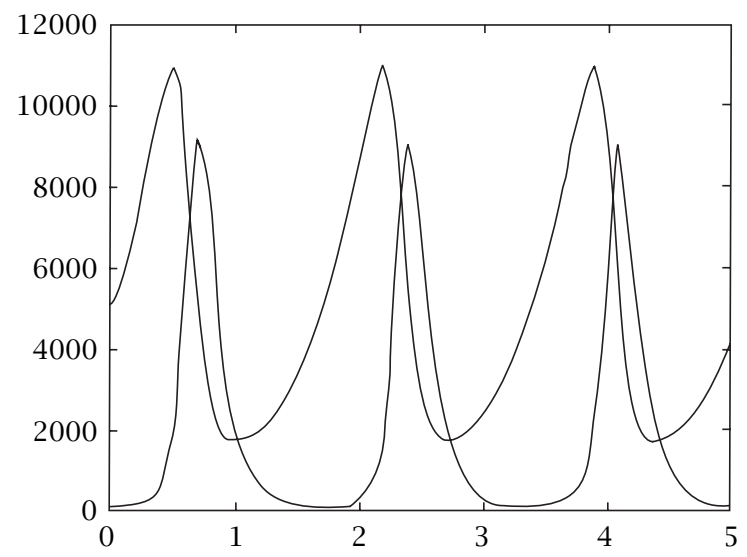

FIGURE 4.1

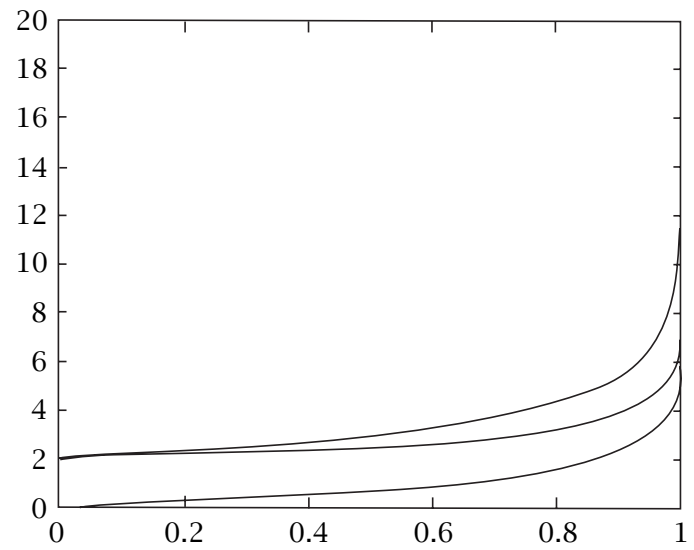

FIGURE 4.2

MODEL 4.2. Ivlev type predator prey system: the system has the form

$$
\frac{d x}{d t}=r x(1-x)-y\left(1-e^{-a x}\right), \quad \frac{d y}{d t}=-D y+s y\left(1-e^{-a x}\right),
$$

where to fit our frame work, $\theta(x)=\left(1-e^{-a x}\right)$ and $s=1$. This system has the only critical point $\left(x^{*}, y^{*}\right)=\left(-\log (1-D) / a,(r / x) x^{*}\left(1-x^{*}\right)\right)$. There are no limit cycles of (4.2) if $0<a \leq 2$ and at most one if $a>2$. In fact if we let

$$
\begin{aligned}
& \gamma(D)=-\frac{(\log (1-D))^{2}}{D+\log (1-D)} \\
& \delta(D)=-\frac{2 D-(1-D) \log (1-D)}{D+(1-D) \log (1-D)} \log (1-D),
\end{aligned}
$$


then for $0<D<1$, we have $2<\gamma(D)<\delta(D)$ and both $\delta(D)$ and $\gamma(D)$ are increasing with respect to $D$. See Figure 4.2. It is also known that (see Sugie [6]) system (4.2) has no limit cycles if either $a \leq \gamma(D)$ or $\gamma(D)<a<\delta(D)$, while the system has a unique stable limit cycle if $a>\delta(D)$.

MODEL 4.3. Predator-prey system of Holling type: (see Sugie [8]); that is,

$$
\begin{aligned}
& \frac{d x}{d t}=r x(1-x)-y \frac{x^{p}}{1-x^{p}}, \\
& \frac{d y}{d t}=-D y+s y \frac{x^{p}}{1-x^{p}},
\end{aligned}
$$

where $\theta(x)=x^{p} /\left(1-x^{p}\right)$ satisfies the assumptions of our setup. It was shown in [8] that it is necessary and sufficient that

$$
(p D-(p-2) s) \sqrt[p]{\frac{D}{s-D}}<(p D-(p-1) s)
$$

for system (4.4) to have a unique limit cycle.

ACKNOWLEDGEMENT. The author would like to thank King Fahd University of Petroleum and Minerals for supporting this research.

\section{REFERENCES}

[1] E. A. Coddington and N. Levinson, Theory of Ordinary Differential Equations, McGraw-Hill, New York, 1955. MR 16,1022b. Zbl 0064.33002.

[2] H. I. Freedman and J. W.-H. So, Global stability and persistence of simple food chains, Math. Biosci. 76 (1985), no. 1, 69-86. MR 87g:92040. Zbl 572.92025.

[3] R. E. Kooij and A. Zegeling, A predator-prey model with Ivlev's functional response, J. Math. Anal. Appl. 198 (1996), no. 2, 473-489. MR 96j:92033. Zbl 851.34030.

[4] Y. Kuang and H. I. Freedman, Uniqueness of limit cycles in Gause-type models of predatorprey systems, Math. Biosci. 88 (1988), no. 1, 67-84. MR 89g:92045. Zbl 642.92016.

[5] G. F. Simmons, Differential Equations with Applications and Historical Notes, International Series in Pure and Applied Mathematics, McGraw-Hill, New York, 1972. MR 58\#17258. Zbl 231.34001.

[6] J. Sugie, Two-parameter bifurcation in a predator-prey system of Ivlev type, J. Math. Anal. Appl. 217 (1998), no. 2, 349-371. MR 98m:92024. Zbl 894.34025.

[7] J. Sugie and T. Hara, Nonexistence of periodic solutions of the Liénard system, J. Math. Anal. Appl. 159 (1991), no. 1, 224-236. MR 92m:34095. Zbl 731.34042.

[8] J. Sugie, R. Kohno, and R. Miyazaki, On a predator-prey system of Holling type, Proc. Amer. Math. Soc. 125 (1997), no. 7, 2041-2050. MR 97m:92005. Zbl 868.34023.

Basem S. Attili: Mathematical Sciences Department, King FaHd University of Petroleum ANd Minerals, DHAHRAN 31261, SAUdi ARABIA

E-mail address: batti1i@kfupm. edu.sa 


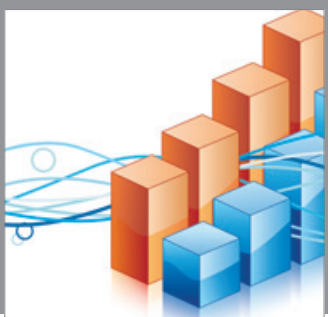

Advances in

Operations Research

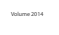

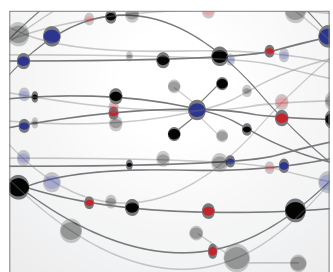

\section{The Scientific} World Journal
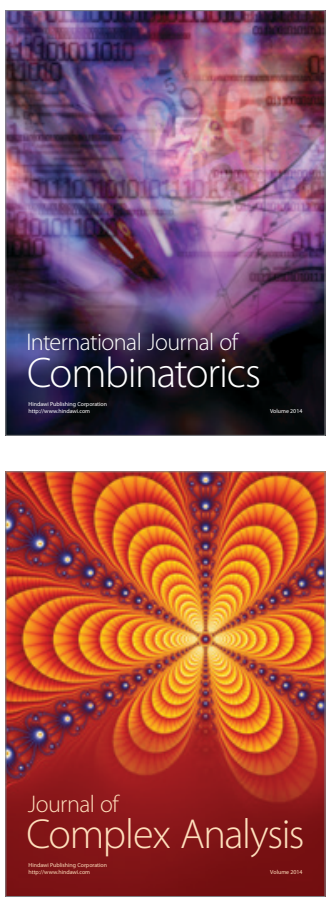

International Journal of

Mathematics and

Mathematical

Sciences
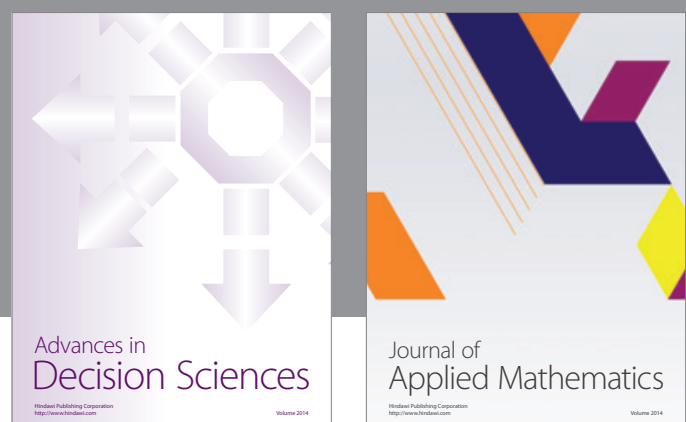

Journal of

Applied Mathematics
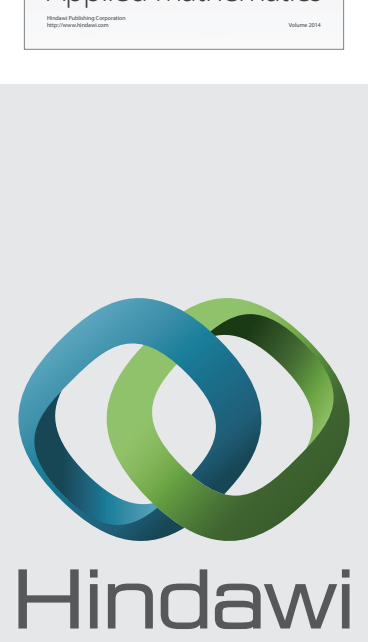

Submit your manuscripts at http://www.hindawi.com
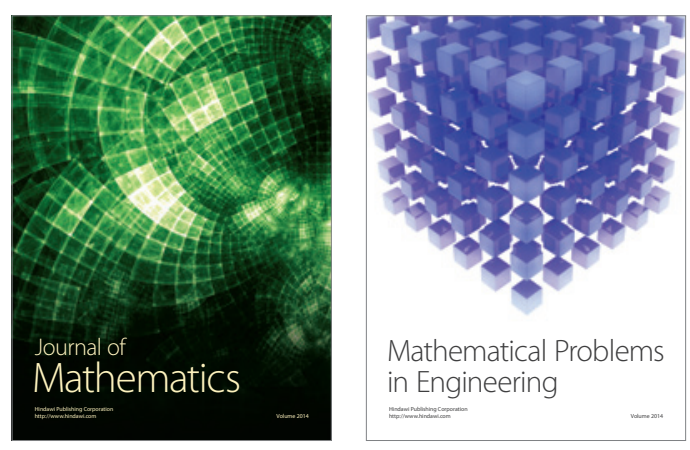

Mathematical Problems in Engineering
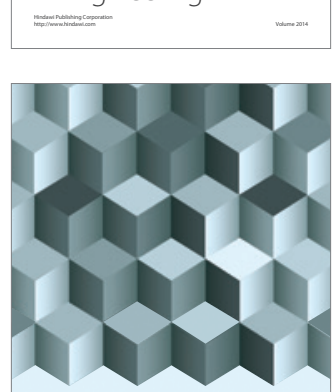

Journal of

Function Spaces
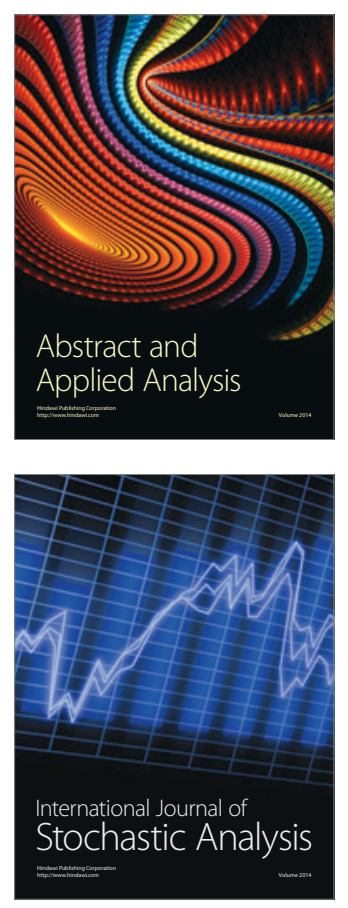

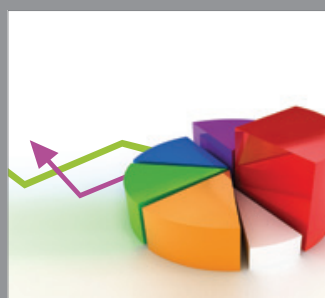

ournal of

Probability and Statistics

Promensencen
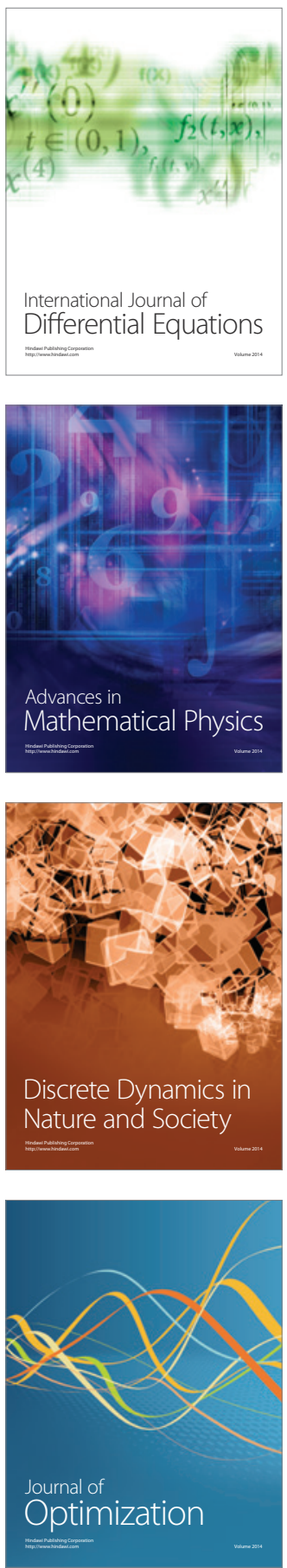